\title{
FATORES ASSOCIADOS AO CONSUMO ALIMENTAR DE IDOSOS RESIDENTES NA REGIÃO SUL DO BRASIL
}

Anny Caroline dos Santos Araujo - Universidade Federal de Ciências da Saúde de Porto Alegre (UFCSPA) -

Email: annycarolinesaraujo@gmail.com

Me. Bruna Senna Rodrigues - CCG Saúde - Email: brusennar@gmail.com

Rozana Ferreira Ortiz - Universidade Federal de Ciências da Saúde de Porto Alegre (UFCSPA) - Email: ro.ortiz1894@gmail.com

Profa. Dra. Valdeni Terezinha Zani - Universidade Federal de Ciências da Saúde de Porto Alegre (UFCSPA)

- Email: valdenizani@ufcspa.edu.br

\section{RESUMO}

Introdução: A Região Sul possui a maior proporção de idosos do país; assim, torna-se necessário avaliar o consumo alimentar desta população visando a elaboração de políticas de alimentação saudável, visto que a má alimentação é um fator de risco importante para Doenças Crônicas Não-transmissíveis, comuns na população idosa. Objetivo: Descrever os fatores associados ao consumo alimentar de idosos residentes no Sul do Brasil. Métodos: Trata-se de uma revisão integrativa da literatura sobre consumo alimentar de idosos da Região Sul, com a busca de artigos publicados de 2015-2020, nas bases Pubmed, Scielo, Bireme e Lilacs. Resultados: Foram selecionados 8 artigos. Observou-se que, os idosos com maior renda e escolaridade tinham padrão alimentar mais saudável, já os idosos de baixa renda tiveram maior consumo de alimentos industrializados, assim como os homens. Houve correlação positiva entre o maior consumo de frutas, verduras e legumes (FVL) em idosos que utilizavam a internet e nas mulheres. Observou-se também, associação de hábitos saudáveis (como não fumar e prática de atividade física) com um padrão saudável de alimentação. Conclusão: A renda e a escolaridade foram os fatores que exerceram maior influência na alimentação dos idosos. Por apresentar grande quantidade de informações sobre alimentação, a internet influenciou positivamente na qualidade de vida deste grupo. $\mathrm{O}$ maior consumo de FVL nas mulheres corrobora com diversos estudos que mostram que a mulher tem hábitos mais saudáveis. Destaca-se a importância do desenvolvimento de estratégias de educação em alimentação e saúde para esta população, principalmente para a população de menor renda.

Palavras-chave: Consumo De Alimentos; Dieta; Idosos. 